\title{
OPEN Actively tunable THz filter based on an electromagnetically induced transparency analog hybridized with a MEMS metamaterial
}

\author{
Ying Huang ${ }^{1}$, Kenta Nakamura ${ }^{2}$, Yuma Takida ${ }^{3}$, Hiroaki Minamide ${ }^{3}$, Kazuhiro Hane ${ }^{2}$ \& \\ Yoshiaki Kanamori ${ }^{1 \bowtie}$
}

Electromagnetically induced transparency (EIT) analogs in classical oscillator systems have been investigated due to their potential in optical applications such as nonlinear devices and the slow-light field. Metamaterials are good candidates that utilize EIT-like effects to regulate optical light. Here, an actively reconfigurable EIT metamaterial for controlling THz waves, which consists of a movable bar and a fixed wire pair, is numerically and experimentally proposed. By changing the distance between the bar and wire pair through microelectromechanical system (MEMS) technology, the metamaterial can controllably regulate the EIT behavior to manipulate the waves around $1.832 \mathrm{THz}$, serving as a dynamic filter. A high transmittance modulation rate of $38.8 \%$ is obtained by applying a drive voltage to the MEMS actuator. The dispersion properties and polarization of the metamaterial are also investigated. Since this filter is readily miniaturized and integrated by taking advantage of MEMS, it is expected to significantly promote the development of $\mathrm{THz}$-related practical applications such as $\mathrm{THz}$ biological detection and $\mathrm{THz}$ communications.

The electromagnetically induced transparency (EIT) effect, resulting from quantum interference, has been extensively researched over the last few decades due to its elimination of absorption in a narrow window within a broad absorption region and its ability to achieve dramatic changes in dispersive properties ${ }^{1-3}$. This phenomenon was later mimicked in nonquantum systems by coupling harmonic oscillators. Specifically, the interference arising from the surface plasmon polariton can be generated by coupling the bright mode, which is directly coupled with the incident electric field, and the dark mode, which is excited by the bright mode ${ }^{4-6}$. Due to the mimicked similarity of the extremely changeable optical response and the rapid change in dispersion in a narrow region, the EIT plasmonic analogy in classical optical systems has garnered increasing attention yearly and can be widely used in the nonlinear ${ }^{7}$, sensor ${ }^{8,9}$, and slow-light ${ }^{10}$ fields. Metamaterials, as artificial optical materials, are a remarkable scheme for realizing a desired EIT plasmonic behavior through the design of a subwavelength structure; their potential has been demonstrated in various applications ranging from antennas ${ }^{11}$ to absorbers ${ }^{12}$, optical switches ${ }^{13}$, and optical buffers ${ }^{14}$ by generating an EIT-like effect. In 2014, Hokari et al. ${ }^{15}$ investigated the different EIT-like phenomena among silver, gold, and aluminum metamaterials consisting of a single wire and a wire pair at visible wavelengths and studied the EIT-like characteristics as functions of the constructional materials as well as the gap distance of the wires. In the same year, this group fabricated plasmonic metamaterials, achieving strong and sharp EIT-like characteristics at wavelengths around $820 \mathrm{~nm}$ with a transmittance of $72 \%{ }^{16}$. In 2015 , Nakamura et al. ${ }^{17}$ successfully demonstrated the EIT-like effect with gold metamaterials comprising a cut wire and a wire pair corresponding to the bright mode and dark mode, respectively.

In recent years, actively controllable EIT metamaterials have been considerably attractive due to the expected realization of dynamic modulation of the transmittance and dispersive characteristics, which is critical for optical practical applications ${ }^{18-20}$. Via several electron injection tuning approaches, such as liquid crystal control, use of the phase transition effect, and use of graphene resonators, regulation of the EIT analog can be achieved due to the tunable modification of optical constants of the medium ${ }^{21-23}$. However, some difficulties exist in the realistic fabrication of these schemes due to the challenges in integrating the tuning triggers for the system. On the other hand, the EIT-like behavior in metamaterials can be manipulated by breaking the symmetry of the

\footnotetext{
${ }^{1}$ Department of Robotics, Tohoku University, Sendai 980-8579, Japan. ${ }^{2}$ Department of Finemechanics, Tohoku University, Sendai 980-8579, Japan. ${ }^{3}$ RIKEN Center for Advanced Photonics, RIKEN, Sendai 980-0845, Japan. ${ }^{\boxplus}$ email: kanamori@meta.mech.tohoku.ac.jp
} 
structure or by varying the structure shape, namely, by regulating the coupling efficiency between the bright and dark resonators, which could be easily managed by microelectromechanical systems (MEMS) in advantageous ways. Moreover, only a small actuation force is required for the MEMS actuation system to work with a high response speed and low power consumption, which allows mechanically reconfigurable metamaterials to be competitive candidates for achieving active EIT-like behavior control $^{24}$. Numerous studies have been conducted to modulate characteristics such as the transmission and spectral dispersion from the visible to near-infrared region based on MEMS metamaterials ${ }^{25-29}$. Liu et al. ${ }^{30}$ experimentally provided an EIT-based metamaterial working in the infrared region, the spectral response of which was obviously modified by inducing structure asymmetry driven by a MEMS actuation system, in which the transmittance for waves at $173 \mathrm{THz}$ changed from below $30 \%$ to above $75 \%$.

Recently, with the ever-increasing attraction of terahertz $(\mathrm{THz})$ waves, which have great potential in communications, imaging, and sensors due to their outstanding performances, such as strong penetration and high resolution $^{31-34}$, metamaterial-based devices that can control $\mathrm{THz}$ waves are being frequently reported ${ }^{35-44}$. In particular, dynamic regulation of $\mathrm{THz}$ waves is highly desired. Therefore, an active filter for manipulating the spectral response of $\mathrm{THz}$ waves is very attractive. Several studies utilizing reconfigurable metamaterials to modify the linewidth of transparency windows in the THz regime based on the EIT-like effect have been proposed ${ }^{45-47}$. However, for some applications, such as biodetectors and spatial communications, selecting a fixed transparency window in the $\mathrm{THz}$ region and altering its transmission properties may be more desirable. Since EIT metamaterials require a trade-off between a small loss and a large dispersion, the design of the reconfigurable EIT structure should carefully strike a balance ${ }^{48}$. In 2012, Gu et al. ${ }^{49}$ first demonstrated active control of an EIT analog at $\mathrm{THz}$ frequencies by using the optically tunable conductivity of the Si islands of a metamaterial on a sapphire substrate, which achieved controllable transmission from 85 to $50 \%$ for $0.74 \mathrm{THz}$ waves, although the high cost and intractability of the sapphire substrate limit the commercialization and miniaturization of the metamaterial. In 2016, Prakash et al. ${ }^{50}$ experimentally proposed an out-of-plane MEMS-driven metamaterial for modulating $\mathrm{THz}$ waves based on the EIT-like effect, for which the transparency peak at $0.55 \mathrm{THz}$ with a transmittance of $70 \%$ controllably dropped while the resonant frequency suffered a shift of $74 \mathrm{GHz}$ as the structure changed. In 2020, Xu et al. ${ }^{51}$ numerically proposed a reconfigurable metamaterial on a silicon substrate to actively filter the waves at $0.65 \mathrm{THz}$ by using an EIT analog. However, all previously reported MEMS metamaterial devices have substrates, and a difficult problem with a substrate is that it creates extra background intensity and interference waveforms, which adversely affect the optical properties of the device. In addition, few works have experimentally demonstrated EIT metamaterials incorporating MEMS to tune the transmittance for particular THz waves.

In this article, an active MEMS-driven metamaterial for manipulating $\mathrm{THz}$ waves at a consistent resonant frequency has been experimentally realized based on the EIT-like effect. The proposed metamaterial consists of a movable bar that functions as a bright-mode resonator and a fixed wire pair that acts as a dark-mode resonator. As the bar is displaced close to the wire pair through an electrostatic force by applying a drive voltage to the MEMS actuator, the transmittance of $\mathrm{THz}$ waves at the resonant frequency is controllably changed. The transmission phase shift associated with the dispersion properties and the polarization of the filter is also investigated. Moreover, the nature of the EIT analog in our metamaterial is analyzed by using numerical calculations and discussed. One of the most important features of this device is the lack of a substrate under the metamaterial. Conventionally, in MEMS metamaterials, the nonplanar bar-pair component that consists of a self-supporting movable part and a substrate-based static part is employed to achieve the tunable EIT-like phenomenon ${ }^{30,52}$, which requires a substrate and thus induces the adverse effect mentioned above. In this work, the bar-pair component was arranged in a plane without a substrate and then successfully fabricated through advanced MEMS fabrication technology. In other words, a high absolute transmittance is obtainable in this self-supporting metamaterial. This is the first time that this type of MEMS metamaterial has been numerically and experimentally demonstrated to our knowledge. In addition, the simple bar-pair shape is used to provide tractability in device fabrication; therefore, a high preparation accuracy of the structure can be feasible, which is significant for generating a strong EIT phenomenon. Moreover, the device is readily miniaturized and integrated into a real optical system by taking advantage of MEMS technology. This filter offers dynamic control of the transmittance and phase of $\mathrm{THz}$ waves, which may greatly promote the development of THz-related practical applications such as $\mathrm{THz}$ medical or biological detection and $\mathrm{THz}$ communications.

\section{Results}

A schematic of the proposed MEMS integrated EIT filter is shown in Fig. 1a, which mainly consists of a gold EIT metamaterial and the mechanical drive system, including high-resistivity silicon supporting bridges and a comb-drive actuator. Each unit cell of the EIT metamaterial (Fig. 1b) is composed of a wire pair fixed on the silicon supporting bridges and a bar stacked on a movable beam that can be displaced by the comb-drive actuator through the electrostatic force. Electrostatic regulation was chosen due to its low power consumption and high tunability ${ }^{24}$. The distance between the bar and the wire pair, denoted g, is initially $30.8 \mu \mathrm{m}$ and is mechanically changeable. When a y-polarized $\mathrm{THz}$ wave is normally incident on the gold metamaterial surface, the bar serves as a dipole resonator, directly coupling with the external light, which corresponds to the bright mode. The wire pair works as a quadrupole resonator with weak interaction with the incident waves, namely, the dark mode. The coupling between these two excitation modes, regarding the EIT analog, is controlled by adjusting the distance between the two resonators, i.e., controlling the lateral displacement of the bar through the mechanical actuator.

An image of the fabricated device was captured by an optical microscope, as shown in Fig. 2 (the fabrication process is illustrated in the Methods section). The close-up views of the unit cell and the mechanical drive system reveal excellent structural sharpness and geometric accuracy. The dimensions of the fabricated EIT metamaterial are very close to the designed values, with a maximum manufacturing error of $0.6 \mu \mathrm{m}$, as shown in Table 1 . The 


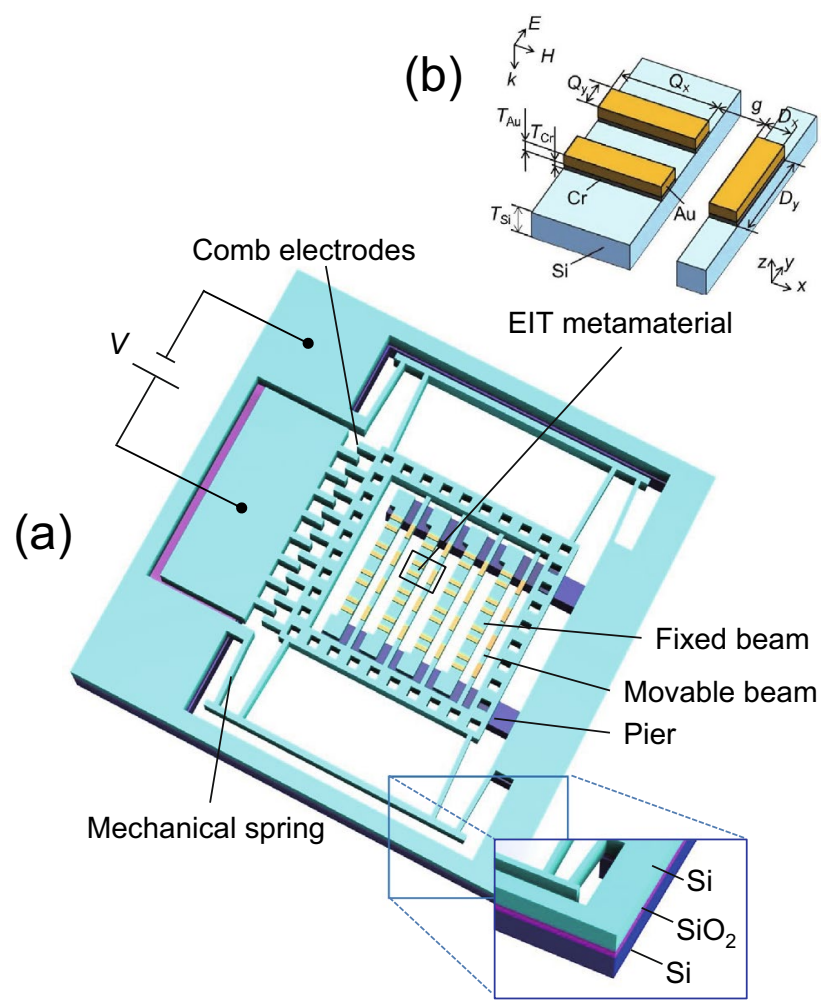

Figure 1. (a) Schematic view of the proposed MEMS integrated device. The close-up view (lower right) shows the overlapping three layers of the SOI wafer. (b) Unit cell of the EIT metamaterial, consisting of a cut wire and a wire pair with the following geometrical parameters: $\mathrm{Q}_{\mathrm{x}}=42.5 \mu \mathrm{m}, \mathrm{Q}_{\mathrm{y}}=10 \mu \mathrm{m}, \mathrm{D}_{\mathrm{x}}=10 \mu \mathrm{m}, \mathrm{D}_{\mathrm{y}}=48.5 \mu \mathrm{m}$, $\mathrm{T}_{\mathrm{Si}}=3.5 \mu \mathrm{m}, \mathrm{T}_{\mathrm{Cr}}=5 \mathrm{~nm}$, and $\mathrm{T}_{\mathrm{Au}}=200 \mathrm{~nm}$. The periods of each unit in both the $\mathrm{x}$ and $\mathrm{y}$ directions are $120 \mu \mathrm{m}$.

(a)

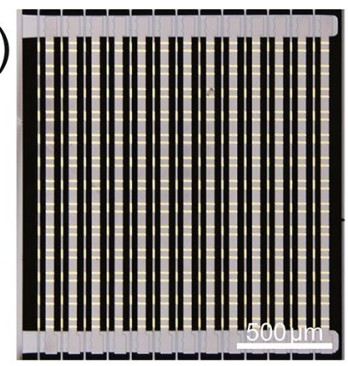

(c)

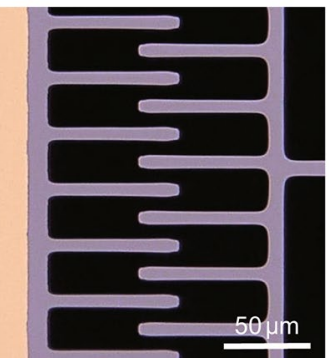

(b)

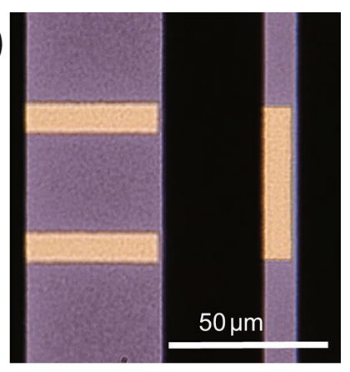

(d)

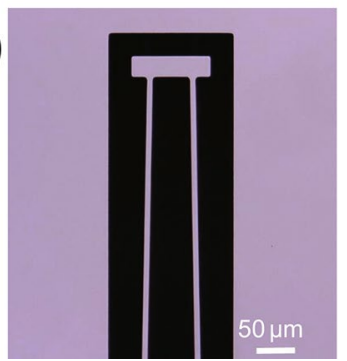

Figure 2. (a) Overview and (b) unit cell of the EIT metamaterial of the fabricated device. Magnified images of the (c) comb drive and (d) mechanical spring of the actuator.

high fabrication accuracy enables the device to achieve strong EIT-like characteristics. A numerical simulation of the modulator was carried out using rigorous coupled-wave analysis (RCWA) based on Maxwell's equations (details of the simulation conditions are shown in the Methods section). The calculated transmittance spectra of the device are plotted in Fig. 3. When the metamaterial is in the initial state with g equal to $30.8 \mu \mathrm{m}$ (see the top image with a black frame in Fig. 3a), the transmittance spectrum of the device presents a deep dip at $1.846 \mathrm{THz}$ 


\begin{tabular}{|l|l|l|l|l|}
\hline & $\mathbf{Q}_{\mathbf{x}}(\boldsymbol{\mu m})$ & $\mathbf{Q}_{\mathbf{y}}(\boldsymbol{\mu m})$ & $\mathbf{D}_{\mathbf{x}}(\boldsymbol{\mu m})$ & $\mathbf{D}_{\mathbf{y}}(\boldsymbol{\mu m})$ \\
\hline Designed value & 42.5 & 10 & 10 & 48.5 \\
\hline Fabricated value & 42.1 & 9.8 & 9.6 & 47.9 \\
\hline
\end{tabular}

Table 1. Comparison between the designed and fabricated values of the metamaterial dimensions.
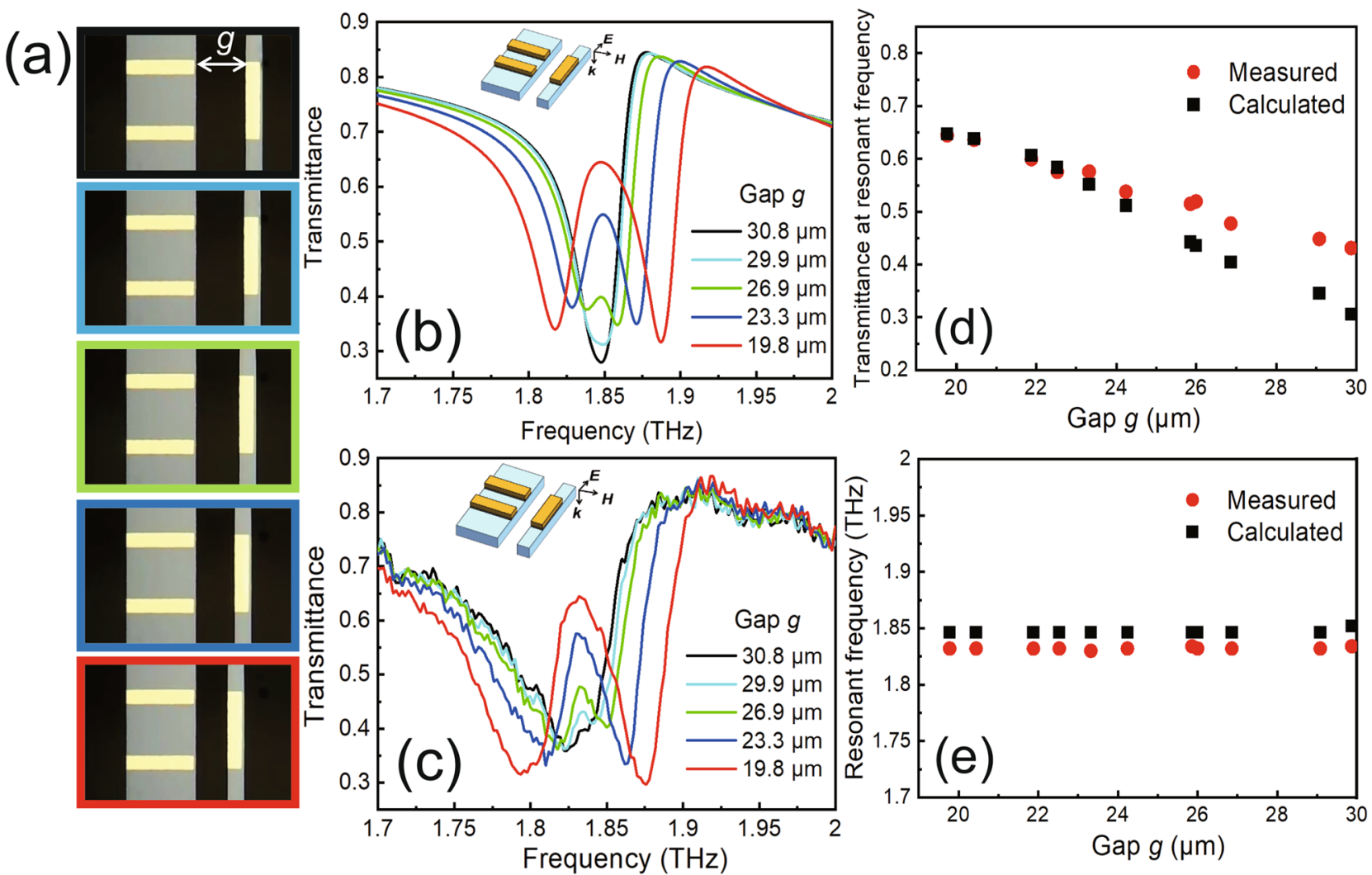

Figure 3. (a) Reconfigurable unit cell of the EIT metamaterial with a resonator gap from 30.8 to $19.8 \mu \mathrm{m}$. (b) Calculated and (c) measured EIT-like transmittance spectra of the device. Comparison of the (d) transmittance at the resonant frequency and (e) resonant frequency between the measured (red dots) and calculated (black squares) results. The images in (a) correspond to the spectra lines of the same color in (b) and (c). The insets in (b) and (c) show the polarization of the incident electric field.

with a minimum transmittance of $27.6 \%$, as shown in Fig. $3 \mathrm{~b}$, which well matches the transmittance spectra of a single bar ${ }^{16}$. This result indicates that the gold bar acting as a dipole antenna is strongly excited by the external field, producing a broadband optical resonance in the transmittance spectrum, whereas the quadruple oscillator does not contribute to the excitation. In other words, the bright mode is dominant, and coupling between the two excitation modes does not occur in the case of a large g. A transmittance peak appears at the resonant frequency of $1.846 \mathrm{THz}$ when $\mathrm{g}$ decreases to $26.9 \mu \mathrm{m}$, which means that the two excitation modes begin to couple due to the close proximity of the dipole and quadruple oscillators. The transmittance peak significantly strengthens and reaches a maximum of $64.7 \%$ as g decreases to $19.8 \mu \mathrm{m}$. The transmittance at the resonant frequency and the resonant frequency as a function of $g$ are shown in Fig. 3d,e, respectively. A high transmittance modulation rate of $57.3 \%$ for $\mathrm{THz}$ waves around $1.846 \mathrm{THz}$ is obtained by the device owing to the EIT-like effect in the metamaterial; here, the modulation rate is defined as $\Delta \mathrm{T} / \mathrm{T}_{\mathrm{Max}}$, where $\mathrm{T}$ is the transmittance at the resonant frequency and $\Delta \mathrm{T}$ is $\mathrm{T}_{\text {Maximum }}-\mathrm{T}_{\text {Minimum. }}$. Note that almost no resonant frequency shift occurs.

Next, the EIT-like phenomenon of the device is demonstrated by measuring the transmittance spectra via a $\mathrm{THz}$ optical measurement system (details of the characteristic system are shown in the Methods section) under various bias voltages. A DC power supply (Keithley Instruments, Model 2410) was used as the voltage source, the back surface of the substrate and the movable comb tooth beam were grounded, and a drive voltage was applied to the fixed supporting bridges. The applied drive voltages of $0,100,150,200$, and $250 \mathrm{~V}$ lead to distances $\mathrm{g}$ of 30.8, 29.9, 26.9, 23.3, and $19.8 \mu \mathrm{m}$, respectively. As shown in Fig. 3c, the classical dipole-yield resonance in the transmittance spectrum with a minimum transmittance of $39.5 \%$ is observed at $1.832 \mathrm{THz}$ without any applied voltage. When the drive voltage increases to $150 \mathrm{~V}$ and g decreases to $26.9 \mu \mathrm{m}$ via electrostatic force actuation, a transparency window clearly appears around the resonant frequency, namely, EIT-like behavior 

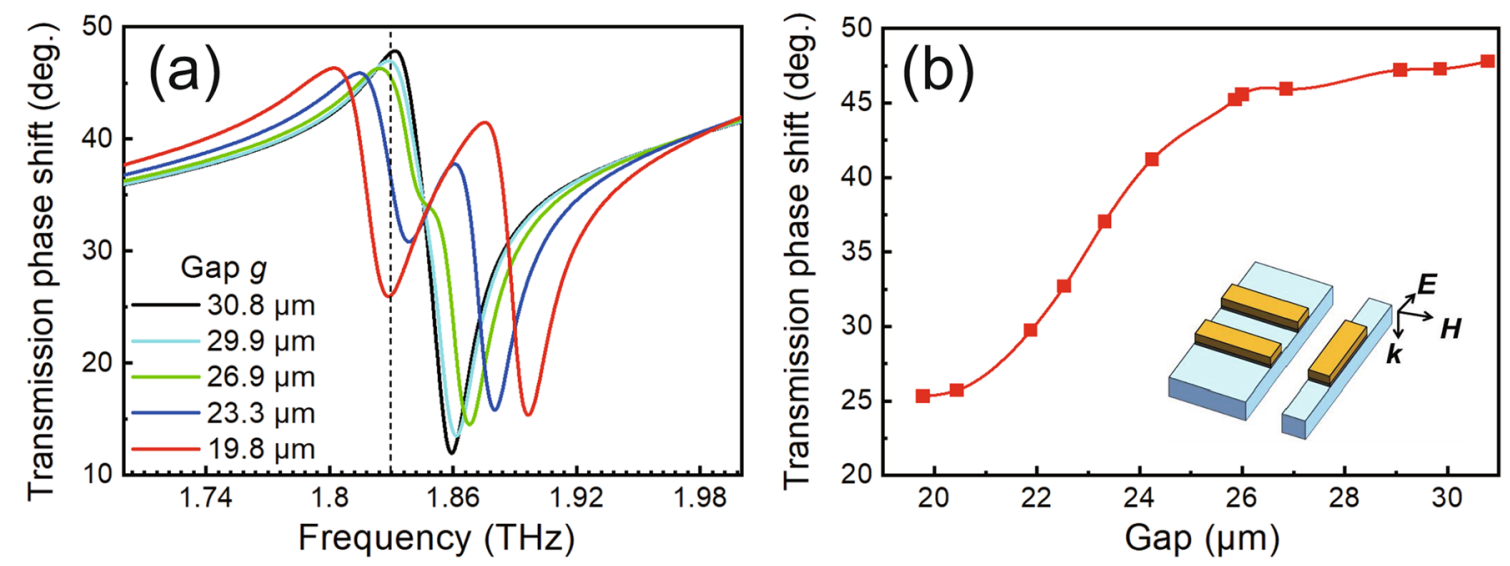

Figure 4. (a) Calculated transmission phase shift of the device with g ranging from 30.8 to $19.8 \mu \mathrm{m}$. The black dashed line shows the resonant frequency position. (b) Relationship between the resonator gap and the transmission phase shift at the resonant frequency.
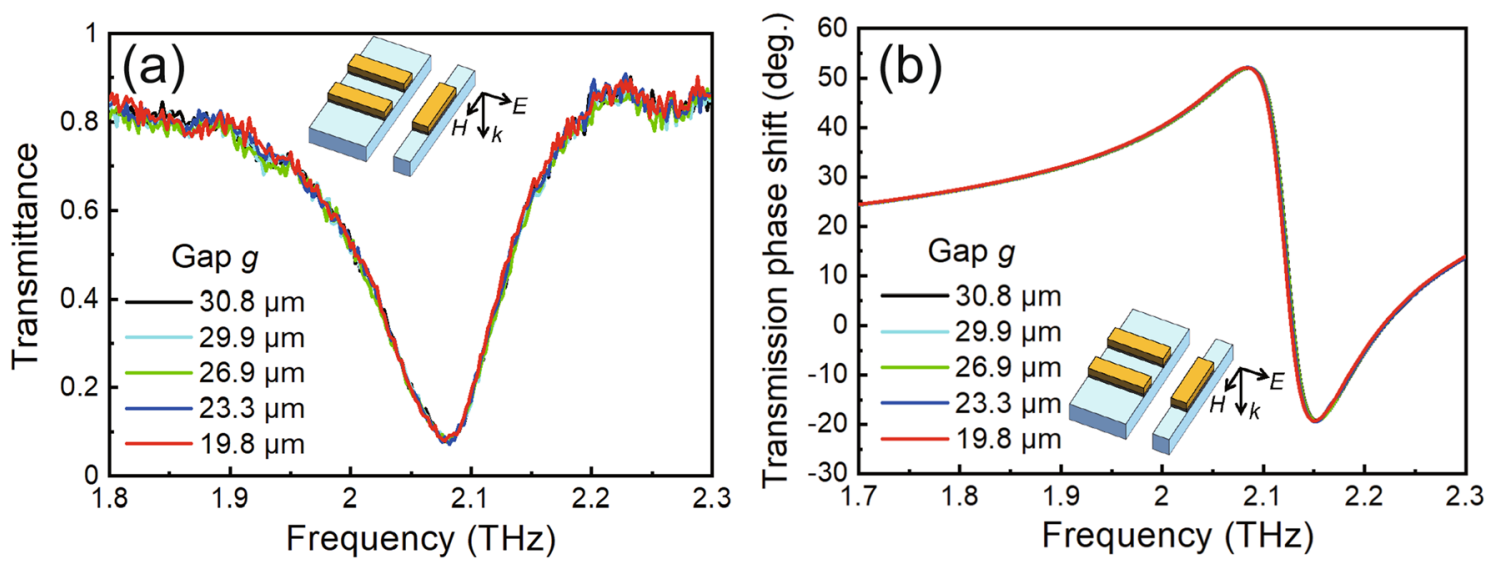

Figure 5. (a) Measured transmittance spectra and (b) transmission phase shift of the device under an incident electric field perpendicular to the cut wire.

occurs. As g dynamically decreases to $19.8 \mu \mathrm{m}$ as the drive voltage increases to $250 \mathrm{~V}$, the modulation depth of the transparency window is controllably enhanced to $64.5 \%$, in good agreement with the calculated results shown in Fig. 3d,e. The device successfully works as an actively tunable filter for waves around $1.832 \mathrm{THz}$ based on the EIT-like behavior via simple adjustment of the applied voltage. As an example, a dynamic modulation demonstration of the fabricated metamaterial in which voltages of 0 and $200 \mathrm{~V}$ are periodically applied to the MEMS actuator to change $\mathrm{g}$ from 30.8 to $23.3 \mu \mathrm{m}$ can be viewed in a Supplementary Video 1. A transmittance modulation rate of $38.8 \%$ for $\mathrm{THz}$ waves around $1.832 \mathrm{THz}$ is experimentally achieved by applying the drive voltage. This performance makes the filter promising for $\mathrm{THz}$ optical switches, $\mathrm{THz}$ communications and other THz-wave-related applications.

Another well-known advantage of EIT analogs is their adjustable dispersion properties, which endow the filter with multifunctionality, such as phase shifting ${ }^{53}$, for $\mathrm{THz}$ waves. Therefore, the transmission phase spectra associated with the dispersion properties of the device were derived from the decline in the real and imaginary parts of the transmission coefficient based on the RCWA method, as plotted in Fig. 4a. The transmission phase shift varies with the $g$ value of the unit cell. At the transparency window frequency of $1.832 \mathrm{THz}$, the phase shift as a function of the g value of the device is plotted in Fig. $4 \mathrm{~b}$. It reaches 47.8 degrees at a $\mathrm{g}$ of $30.8 \mu \mathrm{m}$ and remains almost constant when $g$ is greater than $26 \mu \mathrm{m}$; it then experiences a steep linear drop to 25.3 degrees when $g$ decreases to $19.8 \mu \mathrm{m}$ step by step, which is attributed to the occurrence of coupling between the two excitation modes, well matching the above transmittance results. The dispersion properties of the filter can be dynamically manipulated by MEMS actuation, which may benefit applications in active phase control.

The polarization properties of the device were also investigated by numerical calculations. As Fig. 5a shows, the typical spectral response produced by the dipole resonator instead of the quadruple resonator of the wire pair is observed under $x$-polarized incidence. The bar is unexcited because the external electric field is vertical to it. As g changes from 30.8 to $19.8 \mu \mathrm{m}$, EIT-like behavior does not occur due to the absence of coupling between the dipole and quadruple oscillators, i.e., the absence of an induced reduction in radiation losses. Likewise, the device loses the phase-shift behavior under x-polarized incidence (see Fig. 5b), which shows that this EIT metamaterial serves as a polarization-dependent filter for $\mathrm{THz}$ waves. 


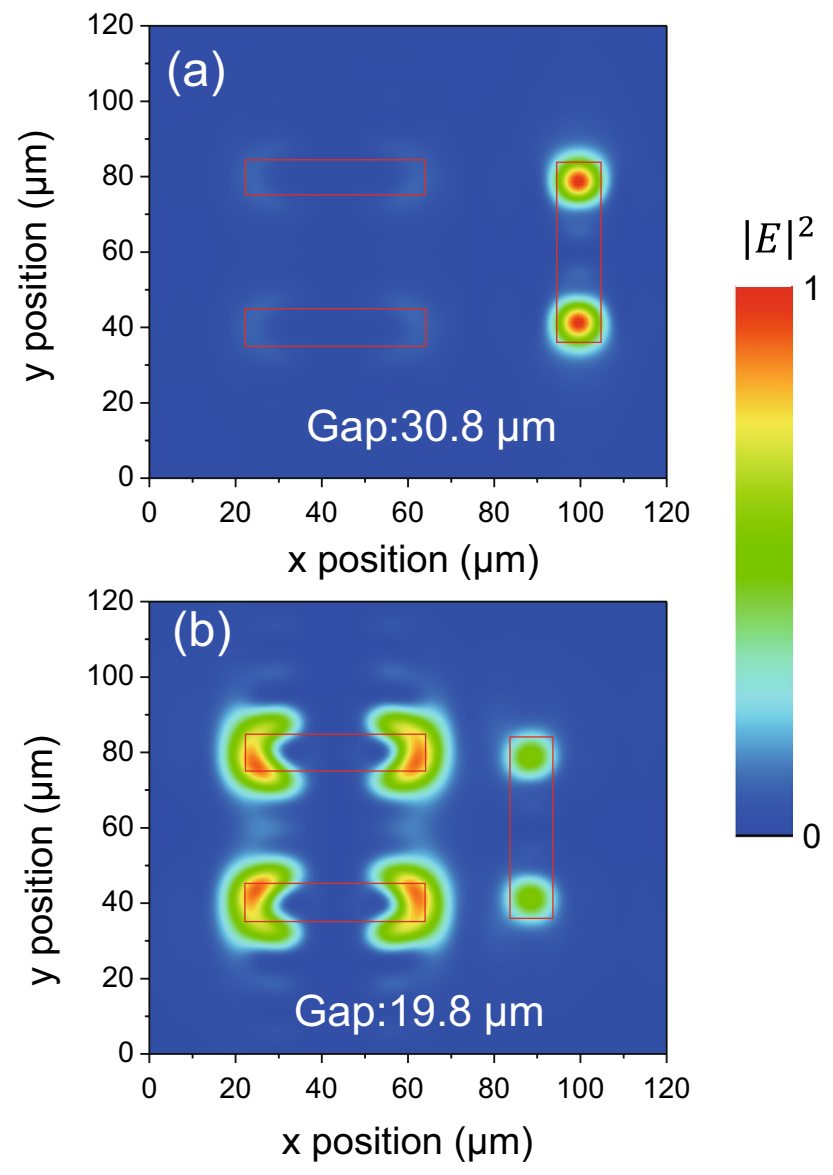

Figure 6. Normalized electric field intensity distributions of the EIT metamaterial unit with a resonator gap of (a) $30.8 \mu \mathrm{m}$ and (b) $19.8 \mu \mathrm{m}$.

Discussion. To further verify the nature of the EIT analog in the proposed device, the electric field intensity distribution on the surface of a unit cell under the on and off states of the EIT phenomenon was calculated and is presented in Fig. 6, which shows the results for a y-polarized wave at a resonant frequency of $1.846 \mathrm{THz}$. For the initial state of the device with g equal to $30.8 \mu \mathrm{m}$ (Fig. 6a), the electric field intensity is concentrated on both ends of the bar, whereas almost none of it is distributed around the wire pair. This observation indicates that a strong bright mode of the bar is directly excited by the external electromagnetic field, contributing to the spectral resonance, and that the dark mode of the quadruple oscillator is completely uncoupled from the bright mode. When the bar and wire pair approach each other with g equal to $19.8 \mu \mathrm{m}$, the dark mode is greatly excited by the bright mode through near field coupling owing to the interference generated between the two modes. Therefore, the electromagnetic energy trapped in the dipole excited mode can be transferred to the quadruple excitation, resulting in localization of the electric field intensity at the ends of the wire pair, as shown in Fig. 6b. Moreover, the significant weakening of the electric field intensity at both ends of the bar reveals the dramatic suppression of the bright mode, namely, the significant suppression of the radiation losses, which leads to the EIT spectral characteristic occurring.

The clear EIT phenomenon reveals the coupling process between the two excitation pathways in the proposed metamaterial: the bright and dark modes excited by the dipole and quadruple oscillators work separately when a large distance exists between the bar and the wire pair, and the dipole antenna suffers very large losses due to the radiative damping caused by the plasmonic transformation and the nonradiative damping arising from the intrinsic metal loss ${ }^{30}$. When the bar moves close to the wire pair, interference between the two oscillators is generated, inducing a strong coupling of the bright and dark modes, which results in suppression of the radiative losses of the dipole oscillator, giving rise to a dramatically enhanced transmittance in a narrow wave range.

Notably, the central resonant frequency of the EIT-like spectra is fixed, whereas the frequencies of the lowfrequency and high-frequency dips exhibit a redshift and a blueshift, respectively, which are observed in both the simulation and measurement results (Fig. 3b,c). Figure 7 shows the calculated transmittance of the device as a function of the gap distance and the incident wavelength. The transparent window (green triangular area within the frame) gradually broadens as the gap decreases, i.e., as the bright-dark mode coupling becomes stronger. In the MEMS metamaterial reported by Liu et al. ${ }^{30}$, a bar-pair component serving as the bright mode and dark mode was used; the EIT-like phenomenon generated in the infrared range had a fixed central resonant frequency, while the frequencies of the two dips shifted following the same trend as that in our work. In the metamaterial reported by Xu et al. ${ }^{51}$, the EIT-like phenomenon was observed in the THz range. The metamaterial consisted of 


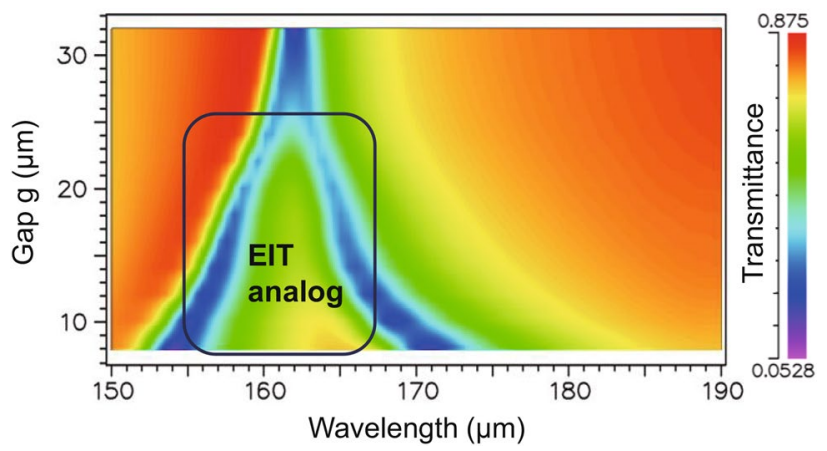

Figure 7. Calculated transmittance of the device as a function of the gap distance and the incident wavelength [drawn with SYNOPSYS DiffractMOD 2020.09-1 (https://www.synopsys.com/ja-jp/japan/products/rsoft-devic e.html)].

a bar and an SRR pair, which were regarded as the bright and dark modes, respectively. By changing the distance between these two modes, the transmittance spectra showed the same dip-shift phenomenon. Unfortunately, the mechanism of the dip shifts in the EIT spectra is still unclear, which is an interesting phenomenon worthy of deep investigation in the near future.

In this work, to obtain high preparation accuracy and sharpness of the metamaterial in fabrication, a simple bar-pair shape with subtly designed geometrical parameters is employed. Moreover, by designing the shape of the metamaterial to achieve multi-coupling modes ${ }^{51}$, transparent multi-windows are possible in our system. Nevertheless, there are a few limitations of this work. Due to the high voltage $(\sim 250 \mathrm{~V})$ requirements of the electrostatic actuator, problems of complex electronic circuits to provide the high voltage and material compatibility arise, which should be improved in the next step.

\section{Conclusions}

In summary, an actively tunable THz filter based on an EIT metamaterial hybridized with MEMS was numerically and experimentally proposed. The metamaterial, with a self-supporting structure, consists of a gold bar and a wire pair without a substrate, which could work without the extra background intensity and interference waveforms that arise from substrates. For y-polarized waves around $1.832 \mathrm{THz}$, the transmittance could be dynamically manipulated to a maximum of $64.5 \%$ with a modulation rate of $38.8 \%$ by controlling the EIT behavior of the proposed filter, namely, by changing the distance between the bar and wire pair, which was successfully demonstrated by applying different drive voltages from 0 to $250 \mathrm{~V}$. In addition, the filter can actively regulate the transmission phase for $1.832 \mathrm{THz}$ waves between 47.8 degrees and 25.3 degrees owing to the tunable dispersive properties. Since the device is readily miniaturized and integrated into a real optical system by taking advantage of MEMS technology, the polarization-dependent filter is significantly favorable for active modulation, switching, memory and other practical applications in the THz wave band.

\section{Method}

Numerical calculation. The numerical calculation was implemented via RCWA, which enables calculation of the exact solutions for periodic structures based on Maxwell's equations. The software SYNOPSYS Diffract MOD with the version number of 2020.09-1 was used to obtain the simulation results. A plane wave light source with an electric field polarized along the bar long axis was used. In both the $\mathrm{x}$ and $\mathrm{y}$ directions of the structure, periodic boundary conditions were set. The order of the Fourier series expansion considered in the simulation was set from -10 to +10 in both the $\mathrm{x}$ and $\mathrm{y}$ directions. The complex refractive indices of $\mathrm{Au}$ and $\mathrm{Cr}$, used as the materials of the metamaterial in the model, were given by D. Rakić et al. ${ }^{54}$.

Fabrication. The fabrication of the EIT metamaterial integrated with MEMS is illustrated in Fig. 8. A cleaned silicon-on-insulator wafer was used (Fig. 8a). First, the top silicon within the metamaterial array area was thinned to a thickness of $3.5 \mu \mathrm{m}$ through photolithography (PMER P-LA900PM photoresist) and inductively coupled plasma-reactive ion etching (ICP-RIE) (Fig. 8b). Second, Cr/Au films with thicknesses of 5/200 nm were deposited on the top Si layer by a sputtering machine, and electrodes and a metamaterial array structure were formed by the ICP-RIE process with a shaped photoresist (PMER P-LA900PM) as a mask (Fig. 8c). Next, photolithography and etching were carried out on the top Si layer to form the comb drive and spring structures for the actuator (Fig. 8d), followed by etching of the backside silicon within the movable area by using a negative resist (MicroChem Co., SU-8 2100) as a mask and ICP-RIE (Fig. 8e). Finally, the $\mathrm{SiO}_{2}$ underneath the etched Si part was sacrificially removed by hydrofluoric acid vapor (SPTS Technologies Ltd., uEtch) to release the actuator and obtain the free-standing structures of the proposed EIT device (Fig. 8f).

Characterization. Figure 9 schematically depicts the utilized optical measurement system ${ }^{55}$. The optical system was set to satisfy the phase matching condition for the pumped light, the injection light, and the terahertz wave (the inset of Fig. 9). MgO: $\mathrm{LiNbO}_{3}$ was used for the nonlinear optical crystal. The collimated THz emissions 
(a) SOI wafer

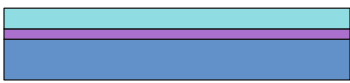

$A$ ताताता

(b) Si base for metamaterial area
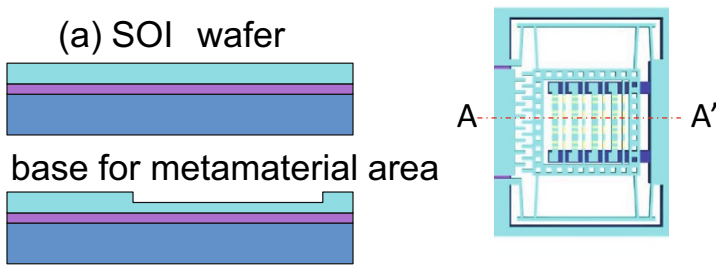

(c) Au structures for metamaterial and electrode

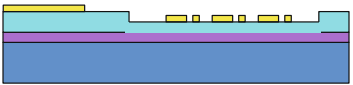

(d) Si etching for Si structure

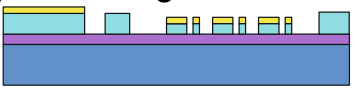

(e) Opening of Si substrate

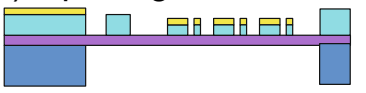

(f) Free-standing structure
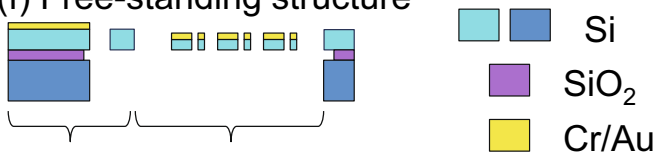

Actuator Metamaterial

Figure 8. Fabrication processes of the MEMS-driven EIT device illustrated in the cross-section profile along A-A'.

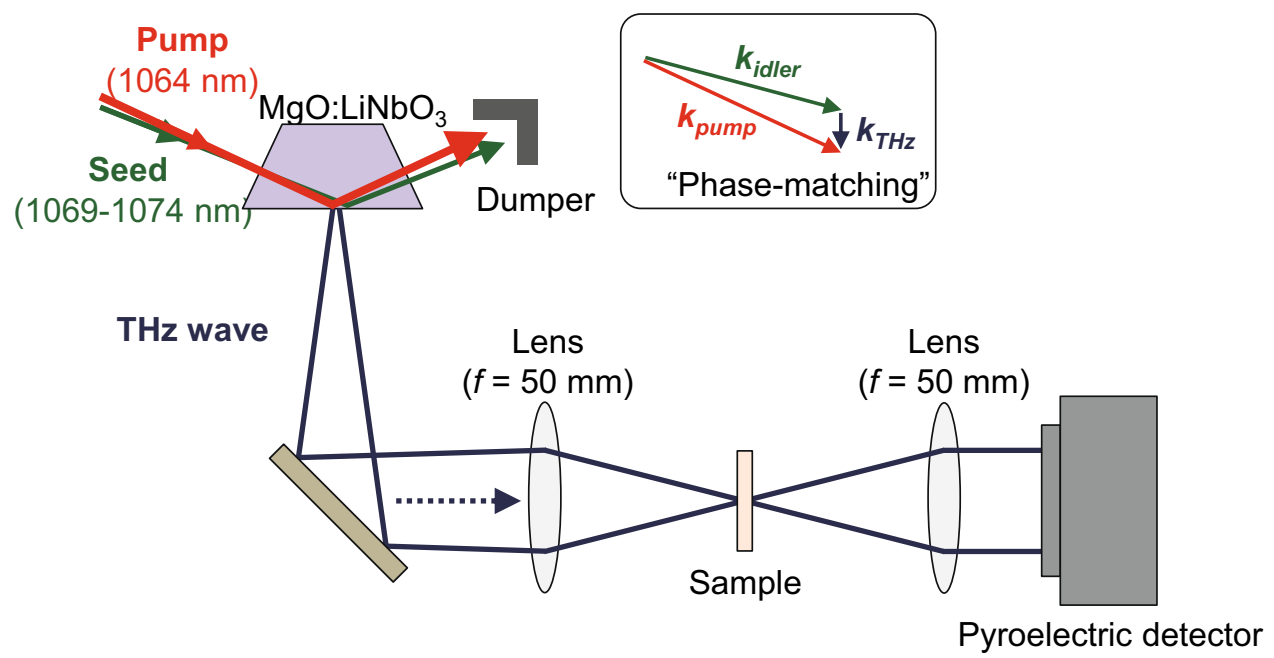

Figure 9. Schematic of the optical measurement system.

were collected by a lens with a focal length of $50 \mathrm{~mm}$ and directed onto the sample, and then, the THz rays that penetrated the sample, carrying information from the sample, were gathered by another lens with a focal length of $50 \mathrm{~mm}$ and output to a pyroelectric detector. The spot diameter of the focused $\mathrm{THz}$ wave was approximately $1.5 \mathrm{~mm}$. The transmittance was averaged by taking four measurements at one point from 1 to $2.5 \mathrm{THz}$ at an interval of $2 \mathrm{GHz}$, which was carried out under a nitrogen atmosphere to avoid the absorption peak of water. All the measured transmittance spectra plotted in this article were the absolute transmittance spectra obtained from the measurement system, which did not undergo normalization. A DC power supply (Keithley Instruments, Model 2410) was employed to realize an adjustable voltage for generating the electrostatic force in the comb drive, which regulates the displacement of the gap in each unit. A maximum displacement of $11 \mu \mathrm{m}$, from an initial gap of 30.8 to $19.8 \mu \mathrm{m}$, was achieved by applying a voltage of $250 \mathrm{~V}$. 
Received: 19 August 2020; Accepted: 18 November 2020

Published online: 30 November 2020

\section{References}

1. Harris, S. E., Field, J. E. \& Imamoĝlu, A. Nonlinear optical processes using electromagnetically induced transparency. Phys. Rev. Lett. 64, 1107-1110 (1990).

2. Fleischhauer, M., Imamoglu, A. \& Marangos, J. P. Electromagnetically induced transparency: optics in coherent media. Rev. Mod. Phys. 77(2), 633 (2005).

3. Boller, K. J., Imamoğlu, A. \& Harris, S. E. Observation of electromagnetically induced transparency. Phys. Rev. Lett. 66(20), 2593 (1991).

4. O’Brien, K. et al. Reflective interferometry for optical metamaterial phase measurements. Opt. Lett. 37(19), 4089-4091 (2012).

5. Garrido Alzar, C. L., Martinez, M. A. G. \& Nussenzveig, P. Classical analog of electromagnetically induced transparency. Am. J. Phys. 70(1), 37-41 (2002).

6. Souza, J. A., Cabral, L., Oliveira, R. R. \& Villas-Boas, C. J. Electromagnetically-induced-transparency-related phenomena and their mechanical analogs. Phys. Rev. A 92(2), 023818 (2015).

7. Harris, S. E. \& Hau, L. V. Nonlinear optics at low light levels. Phys. Rev. Lett. 82, 4611-4614 (1999).

8. Yang, Y., Kravchenko, I. I., Briggs, D. P. \& Valentine, J. All-dielectric metasurface analogue of electromagnetically induced transparency. Nat. Commun. 5(1), 1-7 (2014).

9. Liu, N. et al. Planar metamaterial analogue of electromagnetically induced transparency for plasmonic sensing. Nano Lett. 10, 1103-1107 (2010).

10. Safavi-Naeini, A. H. et al. Electromagnetically induced transparency and slow light with optomechanics. Nature 472(7341), 69-73 (2011).

11. Wang, J. et al. Electromagnetic field manipulation in planar nanorod antennas metamaterial for slow light application. Opt. Commun. 383, 36-41 (2017).

12. Okamoto, K. et al. Electromagnetically induced transparency of a plasmonic metamaterial light absorber based on multilayered metallic nanoparticle sheets. Sci. Rep. 6(1), 1-10 (2016).

13. Vafapour, Z. \& Alaei, H. Achieving a high Q-factor and tunable slow-light via classical electromagnetically induced transparency (Cl-EIT) in metamaterials. Plasmonics 12, 479-488 (2017).

14. Tucker, R. S., Ku, P. C. \& Chang-Hasnain, C. J. Slow-light optical buffers: capabilities and fundamental limitations. J. Lightwave Technol. 23(12), 4046-4066 (2005).

15. Hokari, R., Kanamori, Y. \& Hane, K. Comparison of electromagnetically induced transparency between silver, gold, and aluminum metamaterials at visible wavelengths. Opt. Express 22(3), 3526-3537 (2014).

16. Hokari, R., Kanamori, Y. \& Hane, K. Fabrication of planar metamaterials with sharp and strong electromagnetically induced transparency-like characteristics at wavelengths around $820 \mathrm{~nm}$. J. Opt. Soc. Am. B 31(5), 1000-1005 (2014).

17. Nakamura, K., Hokari, R., Kanamori, Y. \& Hane, K. Fabrication of electromagnetically induced transparency like metamaterials in THz region and evaluation of the transmittance characteristics. IEEJ Trans. Sens. Micromach. 135(11), 454-459 (2015).

18. Zheludev, N. I. \& Kivshar, Y. S. From metamaterials to metadevices. Nat. Mater. 11(11), 917-924 (2012).

19. Billings, L. Exotic optics: metamaterial world. Nat. News 500(7461), 138 (2013).

20. Liu, A. Q., Zhu, W. M., Tsai, D. P. \& Zheludev, N. I. Micromachined tunable metamaterials: a review. J. Opt. 14(11), 114009 (2012).

21. Wang, Y. et al. Tunable optical analog to electromagnetically induced transparency in graphene-ring resonators system. Sci. Rep. 6(1), 1-7 (2016).

22. Xiao, S. et al. Active modulation of electromagnetically induced transparency analogue in terahertz hybrid metal-graphene metamaterials. Carbon 126, 271-278 (2018).

23. Zhou, X., Zhang, T., Yin, X., Chen, L. \& Li, X. Dynamically tunable electromagnetically induced transparency in graphene-based coupled micro-ring resonators. IEEE Photonics J. 9(2), 1-9 (2017).

24. Kanamori, Y., Hokari, R. \& Hane, K. MEMS for plasmon control of optical metamaterials. IEEE J. Sel. Top. Quantum Electron. 21(4), 137-146 (2015).

25. Liu, X. \& Padilla, W. J. Dynamic manipulation of infrared radiation with MEMS metamaterials. Adv. Opt. Mater. 1(8), 559-562 (2013).

26. Hedayati, M. K. et al. Design of a perfect black absorber at visible frequencies using plasmonic metamaterials. Adv. Mater. 23(45), 5410-5414 (2011).

27. Ren, Z. et al. Leveraging of MEMS technologies for optical metamaterials applications. Adv. Opt. Mater. 8(3), 1900653 (2020).

28. Liu, X. \& Padilla, W. J. Thermochromic infrared metamaterials. Adv. Mater. 28(5), 871-875 (2016).

29. Ou, J. Y., Plum, E., Jiang, L. \& Zheludev, N. I. Reconfigurable photonic metamaterials. Nano Lett. 11(5), 2142-2144 (2011).

30. Liu, N. et al. Plasmonic analogue of electromagnetically induced transparency at the Drude damping limit. Nat. Mater. 8(9), 758-762 (2009).

31. Chan, W. L., Deibel, J. \& Mittleman, D. M. Imaging with terahertz radiation. Rep. Prog. Phys. 70(8), 1325 (2007).

32. Nishizawa, S., et al. Terahertz time-domain spectroscopy. in Terahertz Optoelectronics (ed. Sakaki, K.) 203-271 (Springer, Berlin, 2005).

33. Köhler, R. et al. Terahertz semiconductor-heterostructure laser. Nature 417(6885), 156-159 (2002).

34. Pawar, A. Y., Sonawane, D. D., Erande, K. B. \& Derle, D. V. Terahertz technology and its applications. Drug Invention Today 5(2), 157-163 (2013).

35. Wang, B. X., Tang, C., Niu, Q., He, Y. \& Chen, R. A broadband terahertz metamaterial absorber enabled by the simple design of a rectangular-shaped resonator with an elongated slot. Nanoscale Adv. 1(9), 3621-3625 (2019).

36. Wang, B. X. et al. Penta-band terahertz light absorber using five localized resonance responses of three patterned resonators. Res. Phys. 16, 102930 (2020).

37. Wang, B. X., Tang, C., Niu, Q., He, Y. \& Chen, T. Design of narrow discrete distances of dual-/triple-band terahertz metamaterial absorbers. Nanoscale Res. Lett. 14(1), 1-7 (2019).

38. Wang, B. X., Wang, G. Z., Sang, T. \& Wang, L. L. Six-band terahertz metamaterial absorber based on the combination of multipleorder responses of metallic patches in a dual-layer stacked resonance structure. Sci. Rep. 7, 41373 (2017).

39. Wang, B. X. Quad-band terahertz metamaterial absorber based on the combining of the dipole and quadrupole resonances of two SRRs. IEEE J. Sel. Top. Quantum Electron. 23(4), 1-7 (2016).

40. Wang, B. X., He, Y., Lou, P. \& Xing, W. Design of a dual-band terahertz metamaterial absorber using two identical square patches for sensing application. Nanoscale Adv. 2(2), 763-769 (2020).

41. Wang, B. X., Wang, G. Z. \& Wang, L. L. Design of a novel dual-band terahertz metamaterial absorber. Plasmonics 11(2), 523-530 (2016). 
42. Wang, B. X., Wang, G. Z. \& Sang, T. Simple design of novel triple-band terahertz metamaterial absorber for sensing application. J. Phys. D Appl. Phys. 49(16), 165307 (2016).

43. Wang, B. X. et al. A novel dual-band terahertz metamaterial absorber for a sensor application. J. Appl. Phys. 117(1), 014504 (2015).

44. Wang, B. X., Wang, G. Z., Wang, L. L. \& Zhai, X. Design of a five-band terahertz absorber based on three nested split-ring resonators. IEEE Photonics Technol. Lett. 28(3), 307-310 (2015).

45. Sarkar, R. et al. Independently tunable electromagnetically induced transparency effect and dispersion in a multi-band terahertz metamaterial. Sci. Rep. 9(1), 1-10 (2019).

46. Liu, C. et al. Dynamically tunable electromagnetically induced transparency analogy in terahertz metamaterial. Opt. Commun. 410, 17-24 (2018).

47. Yahiaoui, R., Manjappa, M., Srivastava, Y. K. \& Singh, R. Active control and switching of broadband electromagnetically induced transparency in symmetric metadevices. Appl. Phys. Lett. 111(2), 021101 (2017).

48. Zhang, S., Genov, D. A., Wang, Y., Liu, M. \& Zhang, X. Plasmon-induced transparency in metamaterials. Phys. Rev. Lett. 101(4), 047401 (2008).

49. Gu, J. et al. Active control of electromagnetically induced transparency analogue in terahertz metamaterials. Nat. Commun. 3(1), 1151 (2012).

50. Pitchappa, P. et al. Active control of electromagnetically induced transparency analog in terahertz MEMS metamaterial. Adv. Opt. Mater. 4(4), 541-547 (2016).

51. Xu, Y., Wang, X., Chen, X. \& Zhang, L. Structure-based tunable metamaterials for electromagnetically induced transparency windows in low terahertz frequency. J. Appl. Phys. 127(3), 034501 (2020).

52. He, X. J. et al. Dynamic manipulation of electromagnetically induced transparency with MEMS metamaterials. Integr. Ferroelectrics 161(1), 85-91 (2015).

53. Chen, H. T. et al. A metamaterial solid-state terahertz phase modulator. Nat. Photonics 3(3), 148-151 (2009).

54. Rakić, A. D., Djurišić, A. B., Elazar, J. M. \& Majewski, M. L. Optical properties of metallic films for vertical-cavity optoelectronic devices. Appl. Opt. 37(22), 5271-5283 (1998).

55. Takida, Y. et al. Terahertz-wave parametric gain of stimulated polariton scattering. Phys. Rev. A 93(4), 043836 (2016).

\section{Acknowledgements}

The authors acknowledge the financial support of this work by MEXT KAKENHI 16H04342 and 19K22097.

\section{Author contributions}

Y.H. wrote the manuscript, K.N. conducted the research and performed the calculations, Y.T. and H.M. contributed to the THz-wave system development and THz-wave measurement, K.H. gave useful advice in the analysis of the experimental data, and Y.K. planned and supervised the project, prepared the data figures, provided scientific suggestions and revised the manuscript.

\section{Competing interests}

The authors declare no competing interests.

\section{Additional information}

Supplementary information is available for this paper at https://doi.org/10.1038/s41598-020-77922-1.

Correspondence and requests for materials should be addressed to Y.K.

Reprints and permissions information is available at www.nature.com/reprints.

Publisher's note Springer Nature remains neutral with regard to jurisdictional claims in published maps and institutional affiliations.

Open Access This article is licensed under a Creative Commons Attribution 4.0 International License, which permits use, sharing, adaptation, distribution and reproduction in any medium or format, as long as you give appropriate credit to the original author(s) and the source, provide a link to the Creative Commons licence, and indicate if changes were made. The images or other third party material in this article are included in the article's Creative Commons licence, unless indicated otherwise in a credit line to the material. If material is not included in the article's Creative Commons licence and your intended use is not permitted by statutory regulation or exceeds the permitted use, you will need to obtain permission directly from the copyright holder. To view a copy of this licence, visit http://creativecommons.org/licenses/by/4.0/.

(C) The Author(s) 2020 